To APpeAr in ApJ LetTers

Preprint typeset using $\mathrm{AT}_{\mathrm{E} X}$ style emulateapj v. 08/22/09

\title{
FALLING TRANSITING EXTRASOLAR GIANT PLANETS
}

\author{
B. Levrard ${ }^{1}$, C. Winisdoerffer and G. Chabrier \\ Ecole normale supérieure de Lyon, Centre de Recherche Astrophysique de Lyon, \\ Université de Lyon, 46 allée d'Italie, F-69364 Lyon Cedex 07, France \\ To appear in ApJ Letters
}

\begin{abstract}
We revisit the tidal stability of extrasolar systems harboring a transiting planet and demonstrate that, independently of any tidal model, none but one (HAT-P-2b) of these planets has a tidal equilibrium state, which implies ultimately a collision of these objects with their host star. Consequently, conventional circularization and synchronization timescales cannot be defined because the corresponding states do not represent the endpoint of the tidal evolution. Using numerical simulations of the coupled tidal equations for the spin and orbital parameters of each transiting planetary system, we confirm these predictions and show that the orbital eccentricity and the stellar obliquity do not follow the usually assumed exponential relaxation but instead decrease significantly, reaching eventually a zero value, only during the final runaway merging of the planet with the star. The only characteristic evolution timescale of all rotational and orbital parameters is the lifetime of the system, which crucially depends on the magnitude of tidal dissipation within the star. These results imply that the nearly circular orbits of transiting planets and the alignment between the stellar spin axis and the planetary orbit are unlikely to be due to tidal dissipation. Other dissipative mechanisms, for instance interactions with the protoplanetary disk, must be invoked to explain these properties.

Subject headings: celestial mechanics, planetary systems: formation, protoplanetary disks
\end{abstract}

\section{INTRODUCTION}

Jupiter-like extra-solar planets have been detected transiting their parent star at an unexpectedly small distance of less than 0.1 AU (e.g. Pont 2008). Most of these systems have nearly circular orbits (e.g. Pont 2008) and first measurements of the sky-projected angle $\lambda$ between the stellar rotation axis and the planetary orbital axis through the Rossiter-MacLaughlin effect (for HD 209458, HD 149026, HD 189733, TrES-1, XO-1, HD 17156) indicate a nearly perfect spin-orbit alignment (Winn et al. 2005, 2007; Gaudi \& Winn|2007; Narita et al. 2007; Loeillet et al. 2008; Cochran et al. 2008). These observational properties are commonly interpreted as an outcome of tidal dissipation between the host star and the planet and these same effects are also believed to lead to synchronization of the planetary and stellar rotation with the orbital motion. As a consequence, corresponding timescales associated to these processes are usually evaluated by assuming an exponential relaxation towards equilibrium parameters as obtained from any evolution perturbation calculation near an equilibrium state (e.g. Hut 1981). This leads to timescale estimates of spin-orbit alignment, synchronization and circularization which differ by several orders of magnitude, ranging typically from $\sim 10^{5}$ yrs to a Hubble time (e.g. Rasio et al. 1996; Sasselov 2003; Dobbs-Dixon et al. 2004; Ogilvie \& Lin 2008; Mazeh 2008). All these conclusions, however, implicitly assume the existence of such tidal equilibrium states.

It has already been suggested that short-period planets could be unstable to tidal dissipation but these calculations were based on the assumption of the existence of (unstable) tidal equilibrium states (Rasio et al. 1996;

\footnotetext{
1 also at IMCCE-CNRS UMR 8028, 77 Avenue DenfertRochereau, 75014, Paris. blevrard@ens-lyon.fr
}

Dobbs-Dixon et al. 2004), leading to an erroneous application of the tidal stability criterion derived by Hut $(1980)$. More recently, numerical simulations of the orbits of some transiting planets from the OGLE survey indicated a possible collapse with the host star but the effect of tides raised by the star within the planet was ignored (Pätzold et al. 2004; Carone \& Pätzold 2007). Jackson et al. (2008) noticed the importance of considering both tides raised by the star and the planet as well as the non-linear coupled evolution of the eccentricity and the orbital distance, but the global stability of the system and the additional coupling with the rotational evolution were not investigated.

In this Letter, we reconsider the stability of transiting extra-solar planets to tidal dissipation through theoretical and numerical considerations and show that none but one of the transiting planets has a tidal equilibrium state. We investigate the consequences for the tidal evolution timescales of orbital (semi-major axis, eccentricity) and rotational (stellar obliquity, stellar and planetary rotational velocities) parameters, taking both tides raised by the planet and the star into account.

\section{TIDAL STABILITY OF TRANSITING PLANETS}

A binary star-planet system that conserves the total angular momentum $L_{\text {tot }}$ but dissipates its energy is known to dynamically evolve towards only two possible solutions (Counselman 1973; Hut 1980). On one hand, if $L_{\text {tot }}<L_{\mathrm{c}}$, where $L_{\mathrm{c}}$ is the critical angular momentum defined by

$$
L_{\mathrm{c}}=4\left[\frac{G^{2}}{27} \frac{M_{\star}^{3} M_{p}^{3}}{M_{\star}+M_{p}}\left(C_{p}+C_{\star}\right)\right]^{1 / 4},
$$

where $M_{p}, C_{p}$ and $M_{\star}, C_{\star}$ denote the masses and polar moments of inertia of the planet and the star, respec- 
tively, and $G$ is the gravitational constant, no equilibrium state exists and the system ultimately merges, independently of any tidal model. On the other hand, if $L_{\text {tot }}>L_{\mathrm{c}}$, two equilibrium states exist that are characterized by the coincidence between equatorial and orbital planes, circularity of the orbit and synchronization between rotational and orbital periods when no further dissipation occurs (Hut 1980). The furthest equilibrium orbital distance $a_{1}$ is stable while the closest $a_{2}$ is unstable, with $a_{1}>a_{\mathrm{c}}>a_{2}$, where $a_{\mathrm{c}}$ is the marginal equilibrium orbital distance for $L_{\text {tot }}=L_{\mathrm{c}}$, with $a_{\mathrm{c}} \simeq \sqrt{3 C_{\star} / M_{p}} \sim 0.064\left(M_{\mathrm{Jup}} / M_{p}\right)^{1 / 2}$ AU for a planet orbiting a Sun-like star. Therefore, exploring whether a system is stable or not, and deriving characteristic timescales, first requires to find out whether the condition $L_{\text {tot }}<L_{\mathrm{c}}$ is satisfied or not. Neglecting the spin of the planet, the total angular momentum of the system is given by the sum of the orbital angular momentum and the spin of the star:

$$
L_{\mathrm{tot}}=C_{\star} \omega_{\star}+\frac{M_{p} M_{\star}}{\sqrt{M_{p}+M_{\star}}} \sqrt{G a\left(1-e^{2}\right)},
$$

assuming that the stellar obliquity is zero to maximize $L_{\text {tot }}$, where $e$ is the eccentricity, $\omega_{\star}$ is the rotational velocity of the star and $C_{\star}=k M_{\star} R_{\star}^{2}$, where $k$ is set to the typical value 0.06 for centrally condensed stars with dominantly radiative interiors, characteristic of Sun-like stars (Claret \& Gimenez 1989). In order to investigate the fate of observed transiting planetary systems, we have computed the ratio $L_{\mathrm{tot}} / L_{\mathrm{c}}$ for each of them. All the quantities relevant to our study and their uncertainties have been collected from up-to-date published estimates (Table 1 ). The mean value of $L_{\text {tot }} / L_{\mathrm{c}}$ and its standard deviation have been estimated using a Monte-Carlo procedure by sampling the values of $M_{\star}, R_{\star}, M_{p}, a$ and $\omega_{\star}$, considered as normally distributed around their most probable value. When the lower and upper error bars for a variable are not the same, the maximum of the two values has been considered as the standard deviation of the distribution. When only an upper limit of the rotational velocity is available, as for very slowly rotating stars, we considered a flat distribution between 0 and the given upper limit. Finally, we calculated the ratio $L_{\text {tot }} / L_{\mathrm{c}}$ from a random set of the five variables and reproduced this procedure $10^{6}$ times (Fig. 1).

For all but one case, the mean ratio $L_{\text {tot }} / L_{\mathrm{c}}$ is smaller than 1 , indicating that none but one (HAT-P-2b) of the observed transiting planets lies on a tidal equilibrium state and that they will ultimately all fall onto their parent star. Hence, the exponential damping timescale originally put forth by Rasio et al. (1996) (as $a / \dot{a}$ or $e / \dot{e})$ is inappropriate because orbital circularization, synchronization of the spins and spin-orbit alignment do not correspond to the endpoint of tidal evolution. In the following, we define by timescale, the characteristic time required to observe a significant evolution of orbital or rotational parameters.

\section{TIMESCALES FOR THE EVOLUTION OF ORBITAL AND ROTATIONAL PARAMETERS}

In order to determine the characteristic timescales of tidally-unstable extrasolar systems with no asymptotic equilibrium state, we have conducted long-term simu-

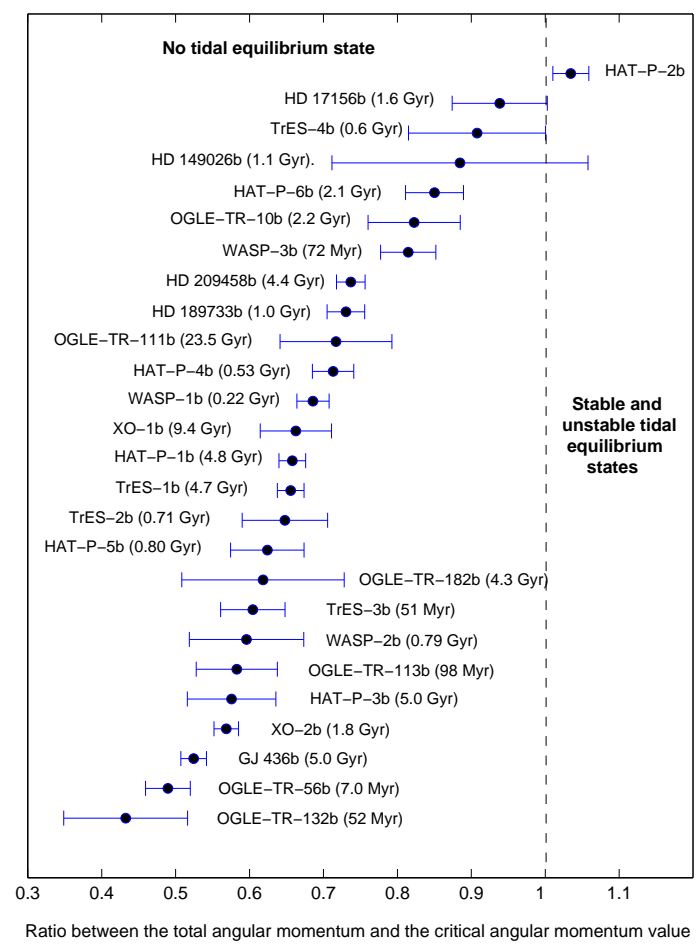

FIG. 1.- List of transiting planets classified by increasing values of the ratio between the total angular momentum of the system $L_{\text {tot }}$ and the critical angular momentum $L_{c}$ (see text). The error bar indicates the standard deviation of the ratio distribution around its mean value. The time remaining for each planet to collide with its host star as obtained from our numerical computations with $Q_{\star}^{\prime}=Q_{p}^{\prime}=10^{6}$ (See Section 3) is indicated between brackets. Note that this time is strongly model-dependent. The HAT-P-2 system is tidally-unstable and very close to the marginally stable regime $a_{1} \simeq a_{c}$. Because its present orbital distance $(\sim 0.068 \mathrm{AU})$ is larger than the stable equilibrium value $\left(a_{1} \sim 0.048 \mathrm{AU}\right)$, the system will reach this state asymptotically.

lations of the fully coupled tidal equations for the orbital and spin parameters, taking tides raised both by the star and the planet into account. We follow the traditional "viscous" approach of the equilibrium tide theory (Darwin 1908) which assumes a constant time lag for any frequency component between the tidally deformed surface of the gaseous envelope and the tidal perturbation, although alternative models are possible given our very limited understanding of tidal processes in gaseous bodies (e.g. Ogilvie \& Lin 2008). The rate of change in the semi-major axis is, at second order in eccentricity (Néron de Surgy \& Laskar 1997)

$$
\begin{aligned}
\frac{d a}{d t}= & \frac{6 M_{p} R_{\star}^{5}}{Q_{\star}^{\prime} M_{\star} a^{4}}\left[\left(1+\frac{27}{2} e^{2}\right) \cos \varepsilon \omega_{\star}-\left(1+23 e^{2}\right) n\right] \\
& +\frac{6 M_{\star} R_{p}^{5}}{Q_{p}^{\prime} M_{p} a^{4}}\left[\left(1+\frac{27}{2} e^{2}\right) \omega_{p}-\left(1+23 e^{2}\right) n\right],
\end{aligned}
$$

where $\omega_{p}$ is the planetary rotation velocity, $\varepsilon$ is the stellar obliquity and $Q_{\star}^{\prime}$ (resp. $Q_{p}^{\prime}$ ) the ratio between the present annual stellar (resp. planetary) tidal quality factor $Q_{\star}$ (resp. $Q_{p}$ ) and the tidal Love number of degree $2 k_{2, \star}$ (resp. $k_{2, p}$ ). The first term in Equation (3) reflects the 

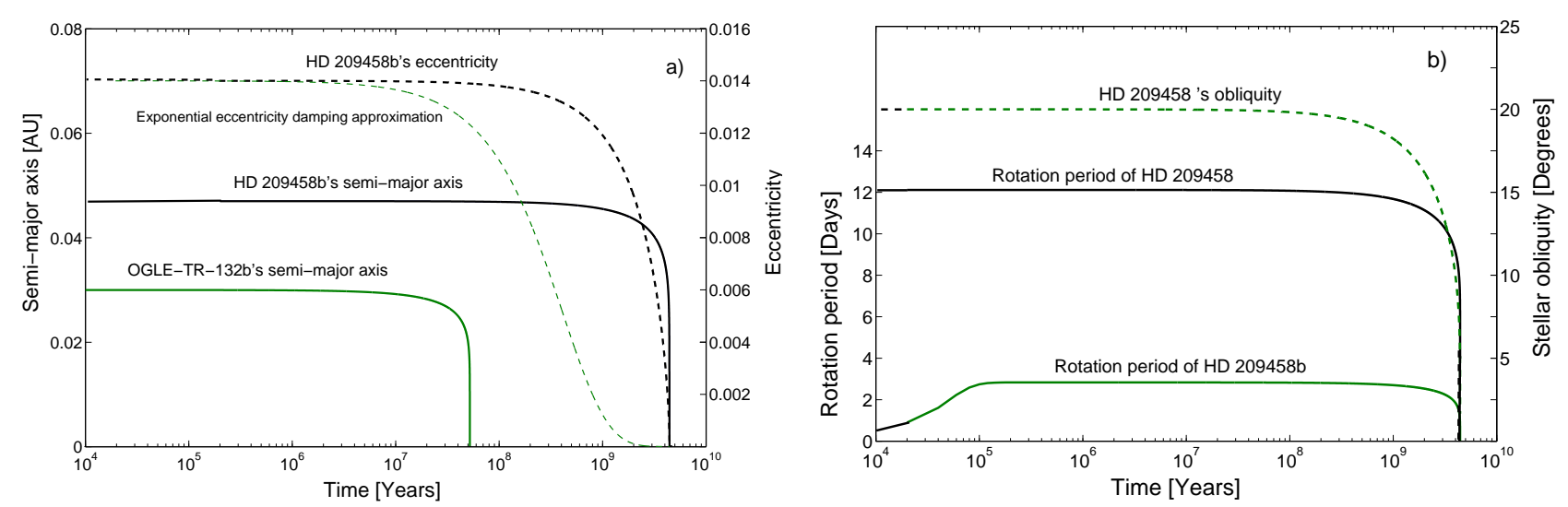

FIG. 2. - Long-term integrations of orbital and rotational parameters of some transiting planets and of their host star. Tidal quality parameters $Q_{\star}^{\prime}$ and $Q_{p}^{\prime}$ are initially set to $10^{6}$ but vary with the semi-major axis as $Q_{\star}=Q_{p}=1 /(n \Delta t)$ where $\Delta t$ is a constant tidal time lag. a) Semimajor axis of OGLE-TR-132b, HD 209458b (solid lines) and eccentricity of HD 209458b (dashed line) as a function of time. The thin dashed line is the usual exponential damping approximation of $e$ with a $4.1 \times 10^{8}$ yr relaxation time assuming that only tides raised on the planet are considered and stable tidal equilibrum states exist ; b) Rotational periods of HD 209458, HD 209458b (solid lines) and HD 209458's obliquity (dashed line) as a function of time. The initial rotational period of HD 209458b and HD 209458 stellar obliquity are arbitrarily set to half a day and $20^{\circ}$ to evaluate the respective timescales of their tidal evolution.

effects of tides within the star while the effects of the tide raised by the star within the planet are reflected in the second term. We assume that the planetary obliquity is zero. Similar equations for the evolution of $e, \varepsilon, \omega_{p}$ and $\omega_{\star}$ can be found in Hut (1981). Since the orbits of transiting planets are nearly edge-on, the angle $\lambda$ is related to the stellar obliquity $\varepsilon$ by $\cos \varepsilon \simeq \cos \lambda \sin i_{\star}$, where $i_{\star}$ is the inclination of the stellar rotation axis relative to the sky plane (Winn et al. 2005). In order to evaluate the typical timescale for the tidal evolution of the projected spin-orbit separation, the initial stellar obliquity was then modified and set to some arbitrary non-zero values. We numerically integrated the complete set of tidal equations forward in time using present values as initial conditions for each transiting planetary system and setting the same initial value $10^{6}$ for $Q_{\star}^{\prime}$ and $Q_{p}^{\prime}$.

The typical evolution of the orbital parameters is shown in Figure 2a for an eccentric planet (HD 209458b) and for the "most unstable" transiting planet OGLETR-132b which lies on a circular orbit. As expected, the evolution of the orbits completely departs from an exponential relaxation, characteristic of the existence of stable or unstable tidal equilibrium states. Both semi-major axis evolve slowly until the planets abruptly collide with their parent star (within $\lesssim 10 \mathrm{Myr}$ and $\lesssim 0.5 \mathrm{Gyr}$, respectively), reaching a zero value in $\sim 52 \mathrm{Myr}$ and $4.3 \mathrm{Gyr}$, respectively. We verified that the same issue holds true for the other unstable transiting planetary systems and the time required for each planet to reach its host star is indicated in Figure 1 for an initial zero stellar obliquity. It typically ranges from $7 \mathrm{Myr}$ (OGLE-TR-56) to 23.5 Gyr (OGLE-TR-111). Similarly (see Fig. 2a), HD 209458 b's eccentricity remains also nearly constant until an ultimate runaway decrease occurs associated to the orbital shrinkage. For comparison, we evaluate for this planet the timescales derived from the usual exponential tidal damping solution, based on the erroneous assumption that transiting planets are close to their tidal equilibrium state : when only tides raised by the star (resp. planet) on the planet (resp. star) are considered, $\tau_{e, p}=(2 / 21) \times\left(Q_{p}^{\prime} / n\right)\left(a / R_{p}\right)^{5}\left(M_{p} / M_{\star}\right) \simeq 0.41 \mathrm{Gyr}$ (resp. $\quad \tau_{e, \star}=(2 / 21) \times\left(Q_{\star}^{\prime} / n\right)\left(a / R_{\star}\right)^{5}\left(M_{\star} / M_{p}\right) \simeq 15$ Gyr), more than one order of magnitude smaller (resp. three times larger) than the afore correctly calculated remaining time before the eccentricity reaches zero.

The evolution of the rotational parameters is illustrated for the HD 209458 system in Figure 2b. Despite the global tidal instability, the planetary rotation velocity is first pseudo-synchronized with the orbital motion $n$ such that $\omega_{p} \sim\left(1+6 e^{2}\right) n$ over the same timescale $\tau_{p s} \sim\left(C_{p} n Q_{p}^{\prime} a^{6}\right) /\left(3 G M_{\star}^{2} R_{p}^{5}\right)$, as the one estimated when tidal equilibrium states exist (Hut 1981). It is close to $\sim 10^{5}$ yr for typical HD 209458b's parameters, much smaller than the orbital decay timescale. This stems from the fact that the angular momentum associated with the planet's rotation is much smaller than the orbital angular momentum. However, Figure 2b clearly shows that it only corresponds to a temporary state because the ultimate orbital collapse causes the orbital mean motion to diverge and then the rotational period of the planet to spin up dramatically. The evolution of HD 209458's rotational period can be easily understood in the framework of classical tidal theory (e.g. Hut 1981; Pätzold et al. 2004; Carone \& Pätzold 2007): because HD 209458 rotates more slowly than its orbital period, the tidal torque raised by the planet onto the star yields the star to spin up with time until a runaway spin-up occurs, due to the conservation of momentum. Hence, HD 209458 is expected to never reach synchronization, contrary to current assumptions. The stellar obliquity follows the same trends and tidal dissipation acts to coplanarize the orbit and the stellar equator only during the final runaway phase of the orbital decay. Figure 2 shows that, except for the existence of a rapid pseudo-synchronization timescale for the planetary rotation, the timescale for the tidal evolution of all the orbital and rotational parameters is the time required for the semi-major axis to shrink to zero, i.e. the remaining lifetime of the system, that has to be evaluated.

There is no simple analytical solution for eqn.(3) but some reasonable simplifications are possible. Indeed, once the planetary spin has rapidly reached its pseudo- 
equilibrium state within $\sim 10^{5}-10^{6}$ yrs, any further exchange of angular momentum between the planet's rotation and its orbit only occurs through radial tides due to a non-zero eccentricity. Assuming that the planet's orbital period is short compared with the star's rotation period, which is true for all unstable transiting planets, eqn.(3) shows that the rate of orbital decay driven by planetary tides becomes smaller than the contribution of tides raised by the planet on the star if the relation:

$$
e<\frac{M_{p}}{M_{\star}} \sqrt{\frac{2 Q_{p}^{\prime}}{7 Q_{\star}^{\prime}}}\left(\frac{R_{\star}}{R_{p}}\right)^{5 / 2}
$$

is verified. This condition is fulfilled for all unstable transiting planetary systems (considering $\left.Q_{p}^{\prime} \simeq Q_{\star}^{\prime}\right)^{2}$. In this context, eqn.(3) can be integrated, giving the remaining time for the planet to reach its host star from its initial orbital distance $a$ (at small eccentricity):

$$
\tau_{a} \simeq \frac{1}{48} \frac{Q_{\star}^{\prime}}{n}\left(\frac{a}{R_{\star}}\right)^{5}\left(\frac{M_{\star}}{M_{p}}\right),
$$

which only depends on tidal dissipation within the star, not within the planet ${ }^{3}$. We stress, however, that such timescales are very uncertain, because of the large uncertainties in the estimate of the $Q_{\star}^{\prime}$ value (typically $10^{5}<Q_{\star}^{\prime}<10^{10}$ ) (e.g. Pätzold et al. 2004). Other tidal models involving dissipation by turbulent viscosity in the convective zone provide estimates of the remaining lifetime several orders of magnitude larger (Rasio et al. 1996; Sasselov 2003; Pätzold et al. 2004). We found the values obtained with eqn.(5) to be in good agreement with those determined from numerical computations for each transiting planet, except for HD 17156 and GJ 436 which have significant eccentricities, leading to a net decrease of the system lifetime due to enhanced tidal interactions. A non-zero stellar obliquity also causes the lifetime of the system to be slightly reduced.

\section{DISCUSSION AND CONCLUSIONS}

Based on rigorous arguments and calculations, we have demonstrated that none but one of the discovered transiting extra-solar planet has a tidal equilibrium state, implying a collapse with their host star. The lack of tidal equilibrium states implies that all the orbital and rotational parameters evolve over the same, only relevant timescale which corresponds to the lifetime of the system. We stress that these results are independent of the tidal model and can probably be extended to most other close-in giant planets detected by radial velocities.

Nevertheless, the exact evaluation of such a timescale strongly depends on the nature of the tidal processes which remain poorly constrained (see e.g. Zahn 1977; Rasio et al. 1996; Sasselov 2003; (Pätzold et al. 2004). The very existence of the currently observed transiting

2 except for GJ 436 for which $\sqrt{2 / 7}\left(M_{p} / M_{\star}\right) \times\left(R_{\star} / R_{p}\right)^{5 / 2} \sim$ $0.08 \lesssim e \sim 0.14$.

3 Using a tidal model for which the quality factor is independent planets suggests that the lifetime of these systems is at least equal to the age of the systems. An alternative possibility is that one or several undetected planetary companions maintain the stability of the present orbit if the planetary orbits are in resonance. Finally, because our results show that both the orbital eccentricity and the stellar obliquity undergo a substantial decay only during the ultimate runaway orbital collapse, this implies that tidal dissipation in the star and in the planet has probably not played a dominant role in the current observations of nearly-circular orbits and spin-orbit alignments of planetary transiting systems, unless each of them is now precisely in this final rapid merging state, a rather unlikely possibility. These results bear important consequences for our understanding of planet formation, migration and planet-disk interaction. They suggest that either the systems were formed with a nearly circular orbit and a stellar spin nearly aligned with the orbital angular momentum, or other processes such as gravitational interactions with the protoplanetary disk have dissipated the initial eccentricity (Moorhead \& Adams 2008). In contrast, planet-planet scattering during the early stages of planet formation (e.g. Chatteriee et al. 2008) or gravitational perturbations by a companion star could have randomized spin-orbit alignment and/or produced large eccentricities (Rasio \& Ford 1996). For sake of simplicity, we have considered that the total angular momentum of the system is constant over time. In reality, angular momentum of the star is continually extracted by a magnetized stellar wind, so that the ratio $L_{\text {tot }} / L_{\mathrm{c}}$ should decrease with time. This indicates that binary systems close to the marginally stable regime like HAT-P-2b (for which $a_{1} \simeq a_{c}$ ) may eventually become unstable to tidal dissipation. One also may argue that tidal dissipation is mostly effective in stellar convective layers (Zahn 1977) so that only the moment of inertia of the outer convective envelope, about $\sim 5 \%$ of the total moment of inertia $C_{\star}$ for a Sun-like star, should be considered both in eqns (1) and (2). In that case, the system convective layers + planet is not isolated because the differential rotation between the convective and radiative zones generates an extra dissipative torque until radiative and convective zones are synchronized. Although this may affect the evolution timescale of the system, the final conclusion should remain the same. We postulate that satellite missions like CoRot or Kepler should detect transiting planets on stable equilibrium orbits, with $L_{t o t}>L_{c}$ and $a>a_{2}$, further away from the tidal instability limit.

The authors are endebted to P. Robutel for useful discussions. This research is supported by the CNRS "Programme National de Planétologie".

of the tidal frequency yields a similar value (the factor $1 / 48$ must be replaced by 2/39) (Pätzold et al. 2004)

\section{REFERENCES}

Carone, L. \& Pätzold, M. 2007, Planet. Space Sci., 55, 643 Chatterjee, S., Ford, E.B., Matsumura, S. \& Rasio, F.A. 2008, ApJ, in press (astro-ph/0703166)
Claret, A. \& Gimenez, A. 1989, A\&A, 37, 81

Cochran, W.D., Redfield, S., Endl, M. \& Cochran, A.L. 2008, ApJ, 683, L59 
Counselman, C.C. 1973, ApJ, 180, 307

Darwin, G. 1908, Scientific Papers, Cambridge University Press, New York, 2

Dobbs-Dixon, I., Lin, D.N.C. \& Mardling, R.A. 2004, ApJ, 610, 464

Gaudi, B.S. \& Winn, J.N. 2007, ApJ, 655, 550

Hut, P. 1980, A\&A, 92, 167

Hut, P. 1981, A\&A, 99, 126

Jackson, B., Greenberg, R. \& Barnes, R. 2008, ApJ, 678, 1396

Loeillet et al. 2008, A\&A, 481, 529

Mazeh, T. 2008, EAS Publications Series 29, Tidal effects in stars, planets and disks, ed. Goupil, M.-J. \& Zahn, J.P., 1

Melo et al. 2006, A\&A, 460, 251

Moorhead, A.V. \& Adams, F.C. 2008, Icarus, 193, 475
Narita et al. 2007, PASJ, 59, 763

Néron de Surgy, O. \& Laskar, J. 1997, A\&A, 318, 975

Ogilvie, G.I. \& Lin, D.N.C. 2008, ApJ, 661, 1180

Pätzold, M., Carone, L. \& Rauer H. 2004, A\&A, 427, 1075

Pont, F. 2008, http://inscience.ch/transits/

Rasio, F.A \& Ford, E. 1996, Science, 274, 954

Rasio, F.A., Tout, C.A., Lubow, S.H., \& Livio, M. 1996, ApJ, 470, L1187

Sasselov, D.D. 2003, ApJ, 596, 1327

Winn et al. 2005, ApJ, 631, 1215

Winn et al. 2007, ApJ, 665, L167

Zahn, J.-P. 1977, A\&A, 57, 383 
Table 1. Values and uncertainties of parameters relevant to tidal evolution of transiting planetary systems

\begin{tabular}{|c|c|c|c|c|c|c|c|c|c|c|c|c|c|c|c|c|}
\hline Name & $M_{p}\left(M_{J}\right)$ & $\Delta M_{p}\left(M_{J}\right)$ & $R_{p}\left(R_{J}\right)$ & $R_{p}^{\min }\left(R_{J}\right)$ & $R_{p}^{\max }\left(R_{J}\right)$ & $T_{\text {orb }}($ days $)$ & $a(A U)$ & $\Delta a(A U)$ & $M_{\star}\left(M_{\odot}\right)$ & $\Delta M_{\star}\left(M_{\odot}\right)$ & $R_{\star}\left(R_{\odot}\right)$ & $\Delta R_{\star}\left(R_{\odot}\right)$ & $v \sin i_{\star}(k m / s)$ & $\Delta v \sin i_{\star}$ & $e$ & $\Delta e$ \\
\hline HD 17156 & $3.12^{\mathrm{c}, d}$ & $0.5^{\mathrm{d}}$ & $1.15^{\mathrm{d}}$ & $1.04^{\mathrm{d}}$ & $1.26^{\mathrm{d}}$ & $21.22^{\mathrm{a}, b}$ & $0.15^{\mathrm{b}}$ & $0.042^{*}$ & $1.2^{\mathrm{a}, b}$ & $0.1^{\mathrm{a}, b}$ & $1.47^{\mathrm{a}, b}$ & $0.17^{\mathrm{c}}$ & $2.6^{\mathrm{c}}$ & $0.5^{\mathrm{c}}$ & $0.67^{\mathrm{c}}$ & $0.08^{\mathrm{c}}$ \\
\hline НаT-P-2 & $8.04^{\mathrm{b}}$ & $0.4^{\mathrm{b}}$ & $0.952^{\mathrm{e}}$ & $0.94^{\mathrm{b}}$ & $1.02^{\mathrm{b}}$ & $5 ? 63341^{\mathrm{a}, b}$ & $0.0677^{\mathrm{a}}$ & $0.0017^{\mathrm{a}}$ & $1.298^{\mathrm{a}}$ & $0.08^{\mathrm{b}}$ & $1.412^{\mathrm{e}}$ & $0.05^{\mathrm{b}}$ & $20.2^{* *}$ & $1.6^{* *}$ & $0.517^{\mathrm{e}}$ & $0.002^{\mathrm{e}}$ \\
\hline HD 149026 & $0.36^{\mathrm{a}, b}$ & $0.03^{\mathrm{a}, b}$ & $0.71^{\mathrm{g}}$ & $0.66^{\mathrm{g}}$ & $0.76^{\mathrm{g}}$ & $2.8766^{\mathrm{f}}$ & $0.042^{\mathrm{f}}$ & $0.000664^{*}$ & $1.3^{\mathrm{a}, \mathrm{b}}$ & $0.06^{\mathrm{b}}$ & $1.45^{\mathrm{a}, b}$ & $0.1^{\mathrm{a}, b}$ & $6.4^{\mathrm{h}}$ & $2.1^{\mathrm{h}}$ & $0.0^{\mathrm{f}}$ & $0.0^{\mathrm{f}}$ \\
\hline HD 189733 & $1.15^{\mathrm{a}, b}$ & $0.04^{\mathrm{b}}$ & $1.154^{\mathrm{b}}$ & $1.137^{\mathrm{b}}$ & $1.171^{\mathrm{b}}$ & $2.218581^{\mathrm{b}}$ & $0.031^{\mathrm{b}}$ & $0.0006^{\mathrm{b}}$ & $0.82^{\mathrm{b}}$ & $0.03^{\mathrm{b}}$ & $0.755^{\mathrm{b}}$ & $0.011^{\mathrm{b}}$ & $3.5^{\mathrm{h}}$ & $1.0^{\mathrm{h}}$ & $0.0^{\mathrm{b}}$ & $0.0^{\mathrm{b}}$ \\
\hline HD 209458 & $0.657^{\mathrm{b}}$ & $0.006^{\mathrm{b}}$ & $1.320^{\mathrm{a}, b}$ & $11.295^{\mathrm{a}, b}$ & $1.345^{\mathrm{a}, b}$ & $3.52474859^{\mathrm{a}, b}$ & $0.047^{\mathrm{b}}$ & $0.0005^{\mathrm{b}}$ & $1.101^{\mathrm{b}}$ & $0.064^{\mathrm{b}}$ & $1.125^{\mathrm{b}}$ & $0.022^{\mathrm{b}}$ & $4.7^{\mathrm{h}}$ & $0.16^{\mathrm{h}}$ & $0.014^{\mathrm{j}}$ & $0.009^{\mathrm{j}}$ \\
\hline GJ 436 & $0.071^{\mathrm{b}}$ & $0.006^{\mathrm{b}}$ & $0.374^{\mathrm{b}}$ & $0.358^{\mathrm{b}}$ & $0.390^{\mathrm{b}}$ & $2.64385^{\mathrm{a}, b}$ & $0.0287^{\mathrm{b}}$ & $0.0003^{\mathrm{b}}$ & $0.44^{\mathrm{b}}$ & $0.04^{\mathrm{b}}$ & $0.463^{\mathrm{b}}$ & $0.02^{\mathrm{b}}$ & $0.52^{* * *}$ & $0.05^{* * *}$ & $0.14^{\mathrm{k}}$ & $0.01^{\mathrm{k}}$ \\
\hline TrES-1 & $0.76^{\mathrm{b}}$ & $0.05^{\mathrm{b}}$ & $1.081^{\mathrm{a}, b}$ & $1.052^{\mathrm{a}, b}$ & $1.110^{\mathrm{a}, b}$ & $3.0300737^{\mathrm{a}, b}$ & $0.0393^{\mathrm{a}, b}$ & $0.0011^{1}$ & $0.89^{\mathrm{b}}$ & $0.035^{\mathrm{b}}$ & $0.811^{\mathrm{b}}$ & $0.020^{\mathrm{b}}$ & $1.08^{\mathrm{n}}$ & $0.3^{\mathrm{n}}$ & $0.0^{\mathrm{b}}$ & $0.0^{\mathrm{b}}$ \\
\hline TrES-2 & $1.198^{\mathrm{b}}$ & $0.053^{\mathrm{b}}$ & $1.220^{\mathrm{b}}$ & $1.178^{\mathrm{b}}$ & $1.265^{\mathrm{b}}$ & $2.47063^{\mathrm{a}, b}$ & $0.0367^{\mathrm{a}, b}$ & $0.001^{\mathrm{m}}$ & $0.98^{\mathrm{a}, b}$ & $0.062^{\mathrm{a}, b}$ & $1.000^{\mathrm{a}, b}$ & $0.036^{\mathrm{a}, b}$ & $2.0^{\mathrm{m}}$ & $1.5^{\mathrm{m}}$ & $0.0^{\mathrm{b}}$ & - \\
\hline TrES-3 & $1.92^{\mathrm{a}, b}$ & $0.23^{a, b}$ & $1.295^{\mathrm{a}, b}$ & $1.214^{\mathrm{a}, b}$ & $1.376^{\mathrm{a}, b}$ & $1.30619^{\mathrm{a}, b}$ & $0.0226^{\mathrm{a}, b}$ & $0.013^{\mathrm{a}}$ & $0.90^{\mathrm{b}}$ & $0.15^{\mathrm{b}}$ & $0.802^{\mathrm{b}}$ & $0.046^{\mathrm{b}}$ & $<2.0^{\mathrm{P}}$ & - & $0.0^{\mathrm{b}}$ & - \\
\hline TrES-4 & $0.84^{\mathrm{a}, b}$ & $0.20^{\mathrm{b}}$ & $1.674^{\mathrm{a}, b}$ & $1.580^{\mathrm{a}, b}$ & $1.768^{\mathrm{a}, b}$ & $3.553945^{\mathrm{a}, b}$ & $0.0488^{\mathrm{a}, b}$ & $0.0022^{\mathrm{a}}$ & $1.22^{\mathrm{b}}$ & $0.17^{\mathrm{b}}$ & $1.738^{\mathrm{b}}$ & $0.092^{\mathrm{b}}$ & $9.5^{\mathrm{q}}$ & $1.0^{\mathrm{q}}$ & $0.0^{\mathrm{b}}$ & - \\
\hline XO-1 & $0.90^{\mathrm{a}, b}$ & $0.07^{\mathrm{a}, b}$ & $1.184^{\mathrm{a}, b}$ & $1.166^{\mathrm{b}}$ & $1.212^{\mathrm{b}}$ & $3.941534^{\mathrm{a}, b}$ & $0.0488^{\mathrm{a}, b}$ & $0.0005^{\mathrm{a}}$ & $1.0^{\mathrm{a}, b}$ & $0.03^{\mathrm{a}, b}$ & $0.928^{\mathrm{a}, b}$ & $0.015^{\mathrm{b}}$ & $<3.0^{\mathrm{r}}$ & - & $0.0^{\mathrm{b}}$ & - \\
\hline HAT-P-1 & $0.53^{\mathrm{a}, b}$ & $0.04^{\mathrm{a}, b}$ & $1.203^{\mathrm{b}}$ & $1.152^{\mathrm{b}}$ & $1.254^{\mathrm{b}}$ & $4.46529^{\mathrm{a}, b}$ & $0.0551^{\mathrm{a}, b}$ & $0.0015^{\mathrm{a}}$ & $1.12^{\mathrm{a}, b}$ & $0.09^{\mathrm{a}, b}$ & $1.115^{\mathrm{b}}$ & $0.043^{\mathrm{b}}$ & $2.2^{\mathrm{s}}$ & $0.2^{\mathrm{s}}$ & $0.09^{\mathrm{s}}$ & $0.02^{\mathrm{s}}$ \\
\hline HAT-P-3 & $0.599^{\mathrm{b}}$ & $0.026^{\mathrm{b}}$ & $0.890^{\mathrm{a}, b}$ & $0.844^{\mathrm{a}, b}$ & $0.936^{\mathrm{a}, b}$ & $2.899703^{\mathrm{a}, b}$ & $0.03894^{\mathrm{a}}$ & $0.0007^{\mathrm{t}}$ & $0.936^{\mathrm{a}, b}$ & $0.062^{\mathrm{a}, b}$ & $0.824^{\mathrm{a}, b}$ & $0.043^{\mathrm{a}, b}$ & $0.5^{\mathrm{t}}$ & $0.5^{\mathrm{t}}$ & $0.0^{\mathrm{b}}$ & - \\
\hline HAT-P-4 & $0.68^{\mathrm{a}, b}$ & $0.04^{\mathrm{a}, b}$ & $1.27^{\mathrm{a}, b}$ & $1.22^{\mathrm{a}, b}$ & $1.32^{\mathrm{a}, b}$ & $3.056436^{\mathrm{a}, b}$ & $0.446^{\mathrm{a}, b}$ & $0.0012^{\mathrm{a}}$ & $1.26^{\mathrm{a}, b}$ & $0.14^{\mathrm{a}, b}$ & $0.59^{\mathrm{a}, b}$ & $0.07^{\mathrm{a}, b}$ & $5.5^{\mathrm{u}}$ & $0.5^{\mathrm{u}}$ & $0.0^{\mathrm{b}}$ & - \\
\hline HAT-P-5 & $1.06^{\mathrm{a}, b}$ & $0.11^{\mathrm{a}, b}$ & $1.26^{\mathrm{a}, b}$ & $1.21^{\mathrm{a}, b}$ & $1.31^{\mathrm{a}, b}$ & $2.788491^{\mathrm{a}, b}$ & $0.04075^{\mathrm{a}}$ & $0.00076^{\mathrm{a}}$ & $1.16^{\mathrm{a}, b}$ & $0.062^{\mathrm{a}, b}$ & $1.167^{\mathrm{a}, b}$ & $0.049^{\mathrm{a}, b}$ & $2.6^{\mathrm{v}}$ & $1.5^{\mathrm{v}}$ & $0.0^{\mathrm{b}}$ & - \\
\hline HAT-P- 6 & $1.057^{\mathrm{a}, b}$ & $0.119^{\mathrm{a}, b}$ & $1.330^{\mathrm{a}, b}$ & $1.269^{\mathrm{a}, b}$ & $1.391^{\mathrm{a}, b}$ & $3.852985^{\mathrm{a}, b}$ & $0.05235^{\mathrm{a}}$ & $0.0087^{\mathrm{a}}$ & $1.29^{\mathrm{a}, b}$ & $0.06^{\mathrm{a}, b}$ & $1.46^{\mathrm{a}, \mathrm{b}}$ & $0.06^{\mathrm{a}, b}$ & $8.7^{\mathrm{w}}$ & $1.0^{\mathrm{w}}$ & $0.0^{\mathrm{b}}$ & - \\
\hline WASP-1 & $0.867^{\mathrm{b}}$ & $0.073^{\mathrm{b}}$ & $1.443^{\mathrm{b}}$ & $1.404^{\mathrm{b}}$ & $1.482^{\mathrm{b}}$ & $2.519961^{\mathrm{a}, b}$ & $0.0382^{\mathrm{a}, b}$ & $0.0013^{\mathrm{a}, b}$ & $1.15^{\mathrm{b}}$ & $0.24^{\mathrm{b}}$ & $1.453^{\mathrm{b}}$ & $0.032^{\mathrm{b}}$ & $5.79^{\mathrm{x}}$ & $0.35^{\mathrm{x}}$ & $0.0^{\mathrm{b}}$ & - \\
\hline WASP-2 & $0.88^{\mathrm{a}}$ & $0.07^{\mathrm{b}}$ & $1.036^{\mathrm{b}}$ & $0.988^{\mathrm{b}}$ & $1.088^{\mathrm{b}}$ & $2.152226^{\mathrm{a}, b}$ & $0.0307^{\mathrm{a}, b}$ & $0.0011^{\mathrm{a}}$ & $0.79^{\mathrm{b}}$ & $0.15^{\mathrm{b}}$ & $0.813^{\mathrm{b}}$ & $0.032^{\mathrm{b}}$ & $<5.0^{\mathrm{y}}$ & - & b & - \\
\hline WASP-3 & $1.76^{\mathrm{a}, b}$ & $0.11^{\mathrm{b}}$ & $1.31^{\mathrm{a}, b}$ & $1.17^{\mathrm{a}, b}$ & $1.38^{\mathrm{a}, b}$ & $1.846834^{\mathrm{a}, b}$ & $0.0317^{\mathrm{a}, b}$ & $0.001^{\mathrm{z}}$ & $1.24^{\mathrm{a}, b}$ & $0.11^{\mathrm{a}, b}$ & $1.31^{\mathrm{a}, b}$ & $0.12^{\mathrm{a}, b}$ & $13.4^{\mathrm{z}}$ & $1.5^{\mathrm{z}}$ & $0.0^{\mathrm{b}}$ & - \\
\hline OGLE-TR-10 & $0.61^{\mathrm{b}}$ & $0.13^{\mathrm{b}}$ & $1.22^{\mathrm{i}}$ & $1.15^{\mathrm{i}}$ & $1.36^{\mathrm{i}}$ & $3.101278^{\mathrm{b}}$ & $0.04162^{\mathrm{a}, b}$ & $0.00069^{\mathrm{B}}$ & $1.10^{\mathrm{b}}$ & $0.05^{\mathrm{b}}$ & $1.14^{\mathrm{b}}$ & $0.11^{\mathrm{b}}$ & $7.0^{\mathrm{A}}$ & $1.0^{\mathrm{A}}$ & $0.0^{\mathrm{b}}$ & - \\
\hline OGLE-TR-56 & $1.29^{\mathrm{a}, b}$ & $0.12^{\mathrm{a}, b}$ & $1.30^{\mathrm{a}, b}$ & $1.25^{\mathrm{a}, b}$ & $1.35^{\mathrm{a}, b}$ & $1.211909^{\mathrm{a}, b}$ & $0.0225^{\mathrm{a}, b}$ & $0.0004^{\mathrm{a}}$ & $1.17^{\mathrm{a}, b}$ & $0.04^{\mathrm{a}, b}$ & $1.32^{\mathrm{a}, b}$ & $0.06^{\mathrm{a}, b}$ & $3.2^{\mathrm{A}}$ & $1.0^{\mathrm{A}}$ & $0.0^{\mathrm{b}}$ & - \\
\hline OGLE-TR-111 & $0.52^{\mathrm{b}}$ & $0.13^{\mathrm{b}}$ & $1.01^{\mathrm{b}}$ & $0.97^{\mathrm{b}}$ & $1.05^{\mathrm{b}}$ & $4.0144479^{\mathrm{a}, b}$ & $0.07^{\mathrm{C}}$ & $0.001^{\mathrm{C}}$ & $0.81^{\mathrm{b}}$ & $0.02^{\mathrm{b}}$ & $0.831^{\mathrm{a}}$ & $0.031^{\mathrm{a}}$ & $<5.0^{\mathrm{a}}$ & - & $0.0^{\mathrm{b}}$ & - \\
\hline OGLE-TR-113 & $1.32^{\mathrm{a}, b}$ & $0.19^{\mathrm{a}, b}$ & $1.09^{\mathrm{a}, b}$ & $1.06^{\mathrm{a}, b}$ & $1.12^{\mathrm{a}, b}$ & $1.4324757^{\mathrm{a}, b}$ & $0.0229^{\mathrm{a}, b}$ & $0.0002^{\mathrm{a}}$ & $0.78^{\mathrm{a}, b}$ & $0.02^{a, b}$ & $0.77^{\mathrm{a}, b}$ & $0.02^{\mathrm{a}, b}$ & $<5.0^{\mathrm{A}}$ & - & $0.0^{\mathrm{b}}$ & - \\
\hline OGLE-TR-132 & $1.14^{\mathrm{a}, b}$ & $0.12^{\mathrm{a}, b}$ & $1.18^{\mathrm{a}, b}$ & $1.11^{\mathrm{a}, b}$ & $1.25^{\mathrm{a}, b}$ & $1.689868^{\mathrm{a}, b}$ & $0.0306^{\mathrm{D}}$ & $0.0008^{\mathrm{D}}$ & $1.26^{\mathrm{a}, b}$ & $0.03^{\mathrm{a}, b}$ & $1.34^{\mathrm{a}, b}$ & $0.08^{\mathrm{a}, b}$ & $<5.0^{\mathrm{A}}$ & - & $0.0^{\mathrm{b}}$ & - \\
\hline OGLE-TR-182 & $1.01^{\mathrm{a}, b}$ & $0.15^{\mathrm{a}, b}$ & $1.13^{\mathrm{a}, b}$ & $1.05^{\mathrm{a}, b}$ & $1.37^{\mathrm{b}}$ & $3.97910^{\mathrm{a}, b}$ & $0.051^{\mathrm{a}, b}$ & $0.001^{\mathrm{a}}$ & $1.15^{\mathrm{a}, b}$ & $0.05^{\mathrm{a}, b}$ & $1.15^{\mathrm{a}, b}$ & $0.23^{\mathrm{a}, b}$ & $<5.0^{\mathrm{E}}$ & - & $0.0^{\mathrm{b}}$ & - \\
\hline
\end{tabular}

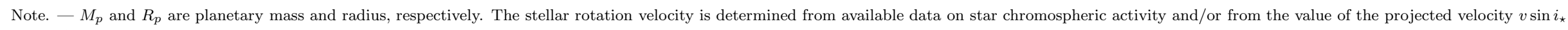
obtained from Doppler spectroscopic measurements, where $i_{\star}$ is the angle between the stellar rotation axis and the line-of-sight, that we assumed to be close to $\simeq 90^{\circ}$.

*estimated via Kepler's law

**based on the average of Loeillet et al. (2008), Bakos et al. (2007) and Winn et al. (2007)

***estimated via chromospheric activity $(\simeq 45$ days by Demory et al. 2007$)$ 